\title{
Orbital-dependent Fermi surface shrinking as a fingerprint of nematicity in FeSe
}

\author{
Laura Fanfarillo, ${ }^{1,}=$ Joseph Mansart, ${ }^{2,}=$ Pierre Toulemonde, ${ }^{3,4}$ Hervé Cercellier,,${ }^{3,4}$ Patrick Le Fèvre, ${ }^{5}$ \\ François Bertran, ${ }^{5}$ Belen Valenzuela, ${ }^{6}$ Lara Benfatto, ${ }^{7}$ and Véronique Brouet ${ }^{2, *}$ \\ ${ }^{1}$ CNR-IOM and International School for Advanced Studies (SISSA), Via Bonomea 265, I-34136 Trieste, Italy \\ ${ }^{2}$ Laboratoire de Physique des Solides, CNRS, Univ. Paris-Sud, Université Paris-Saclay, 91405 Orsay Cedex, France \\ ${ }^{3}$ Université Grenoble Alpes, Institut NEEL, F-38000 Grenoble, France \\ ${ }^{4}$ CNRS, Inst. NEEL, F-38000 Grenoble, France \\ ${ }^{5}$ Synchrotron SOLEIL, L'Orme des Merisiers, Saint-Aubin-BP 48, 91192 Gif sur Yvette, France \\ ${ }^{6}$ Instituto de Ciencia de Materiales de Madrid, ICMM-CSIC, Cantoblanco, E-28049 Madrid, Spain \\ ${ }^{7}$ ISC-CNR and Department of Physics, "Sapienza" University of Rome, Piazzale Aldo Moro 5, 00185, Rome, Italy
}

(Received 18 July 2016; revised manuscript received 23 September 2016; published 21 October 2016)

\begin{abstract}
A large anisotropy in the electronic properties across a structural transition in several correlated systems has been identified as the key manifestation of electronic nematic order, breaking rotational symmetry. In this context, FeSe is attracting tremendous interest, since electronic nematicity develops over a wide range of temperatures, allowing accurate experimental investigation. Here we combine angle-resolved photoemission spectroscopy and theoretical calculations based on a realistic multiorbital model to unveil the microscopic mechanism responsible for the evolution of the electronic structure of FeSe across the nematic transition. We show that the self-energy corrections due to the exchange of spin fluctuations between hole and electron pockets are responsible for an orbital-dependent shrinking of the Fermi surface that affects mainly the $x z / y z$ parts of the Fermi surface. This result is consistent with our experimental observation of the Fermi surface in the high-temperature tetragonal phase, which includes the $x y$ electron sheet that was not clearly resolved before. In the low-temperature nematic phase, we experimentally confirm the appearance of a large $(\sim 50 \mathrm{meV}) x z / y z$ splitting. It can be well reproduced in our model by assuming a moderate splitting between spin fluctuations along the $x$ and $y$ crystallographic directions. Our mechanism shows how the full entanglement between orbital and spin degrees of freedom can make a spin-driven nematic transition equivalent to an effective orbital order.
\end{abstract}

DOI: 10.1103/PhysRevB.94.155138

\section{INTRODUCTION}

Electronic nematic phases are ordered states, where electrons spontaneously break the rotational point-group symmetry of the crystal, but not its translational symmetry. They are increasingly believed to play an important role in many correlated systems [1]. In recent years, iron-based superconductors have provided remarkable examples for such behaviors [2-4]. Indeed, the electronic properties manifest a much larger anisotropy across the tetragonal to orthorhombic transition than expected from the structural changes alone. One possibility is that the nematic phase is a precursor of the antiferromagnetic order that usually emerges at lower temperature by selecting an ordering wave vector along the $x$ direction. However, direct measurements of the band structure seem to point to a true symmetry-breaking state with a charge unbalance between the $x z$ and $y z$ orbitals. As these degrees of freedom are strongly entangled, it is not easy to discriminate their respective role.

FeSe offers the unique opportunity to study the nematic behavior occurring below the structural transition at $T_{S} \sim 90 \mathrm{~K}$ in the absence of any long-range magnetic ordering [5]. A superconducting state eventually develops below $9 \mathrm{~K}$ in bulk FeSe samples [6]. In this wide temperature range, the system shows a marked electron nematicity in transport [7]. Angle-resolved photoemission spectroscopy (ARPES)

\footnotetext{
$=$ L.F. and J.M. contributed equally to this work.

*Corresponding author: veronique.brouet@u-psud.fr
}

investigations [8-12] have revealed a $50 \mathrm{meV}$ splitting at the $M$ point of the Brillouin zone (BZ) between $x z$ and $y z$ orbitals. However, this splitting is different at $\Gamma[11,12]$ and actually of opposite sign [13]. This rules out a simple on-site unbalance of orbital occupation [14] (ferro-orbital order) and suggests instead the emergence of momentummodulated orbital ordering [15-17], whose microscopic origin still remains debated. Even though the spin-driven nematic scenario was first considered as unlikely due to the lack of long-range magnetic order or precursor effects [18,19], more recently sizable spin fluctuations have been detected [20-23]. In addition, it has been pointed out that the absence of longrange magnetic order could be due to frustration [24], leaving open the possibility that fluctuating magnetism can play a role also in FeSe, as it occurs in other iron-based systems.

In addition to the above findings, FeSe exhibits, already at high temperatures well above $T_{S}$, a dramatic "shrinking" of the Fermi surface (FS) pockets as compared to local density approximation calculations. This means that bands are shifted in opposite downward/upward directions for hole pockets at $\Gamma$ and electron pockets at $M$, respectively. This has been observed previously in a number of iron pnictides, by quantum oscillations [25] or ARPES [26], but is rarely considered as an important fingerprint of interactions in these systems. Dynamical mean-field theory (DMFT) calculations for example reproduce remarkably well the observed mass renormalizations in these systems [27,28], but they do not predict a strong shrinking in general, and not in FeSe [28,29]. On the other hand, the exchange of spin fluctuations between hole and electron pockets can provide a general mechanism for 
the FS shrinking in pnictides [30,31]. In this paper, we extend this previous approach [30] to a realistic microscopic model for spin interactions where the spin-fluctuation exchange mechanism is orbital selective, as recently pointed out in Ref. [32]. We then argue that an orbital-dependent shrinking of the Fermi surfaces is the key mechanism to understand the nematic transition in FeSe. Experimentally, we achieve a clear identification in ARPES measurements of the $x y$ electron band. This allows us to establish that above $T_{S}$ the FS shrinking is stronger than in other pnictides and it is orbital selective, the $x z / y z$ FS sheets being much more severely affected than the $x y$ sheets. Below $T_{S}$, the $x y$ electron band is basically unaffected, supporting the original [8-12] view-questioned in some recent reports $[33,34]$ - that the band-structure modifications arise from a $50 \mathrm{meV}$ splitting of the $x z-y z$ orbitals. This energy splitting of the $x z / y z$ orbitals directly follows from an orbital differentiation of the FS shrinking mechanism. At microscopic level this is due to the anisotropy of the spin fluctuations peaked at ordering vectors along $k_{x}$ or $k_{y}$, a fingerprint of the spin-driven nematic scenario [3]. Our picture not only creates a strong link between the FS shrinking and nematicity, able to describe the ARPES data above and below the structural transition, but it also solves the apparent dichotomy between spin-driven and orbital-driven nematic scenarios, which merge in our approach in a unified orbital-selective spin-fluctuation nematic mechanism.

\section{EXPERIMENTAL DETAILS}

Single crystals have been grown using the chemical vapor transport method in sealed quartz tube, starting from $\mathrm{Fe}$ and Se powders (with a 1.1:1 molar ratio) in a eutectic $\mathrm{KCl}+\mathrm{AlCl}_{3}$ chlorides mixture. Details and characterization can be found in Ref. [35]. The observation of quantum oscillations [36] attests from the samples' quality.

ARPES measurements were carried out at the CASSIOPEE beamline of the SOLEIL synchrotron, with a Scienta R4000 analyzer, an angular resolution of $0.3^{\circ}$, and an overall energy resolution better than $10 \mathrm{meV}$. The measurements in Figs. 2 and 4 were carried out at a photon energy of $40 \mathrm{eV}$ with linear polarization along $k_{x}$. This selects even orbitals along $k_{x}$ and odd orbitals along $k_{y}$. More details are given in the Supplemental Material (SM) [37].

\section{RESULTS AND DISCUSSION}

\section{A. Model of orbital-selective shrinking}

As a starting point, we model the band structure with the tight-binding model of Ref. [38] [Fig. 1(a)], where the renormalization of the bands due to Hubbard and Hund's like interactions is already taken into account. As will be justified by our ARPES data later on, this requires a high-temperature renormalization of 3 for $x z / y z$ and 5 for $x y$ orbitals, in good agreement with DMFT calculations [27,28]. To allow for an analytical treatment we map this dispersion into a low-energy two-dimensional model able to describe the relevant orbital content of the pockets around the $\Gamma, M_{X}(\pi, 0)$, and $M_{Y}$ $(0, \pi)$ points in the $1 \mathrm{Fe} \mathrm{BZ}$ [39]. The Hamiltonian at each point can be represented as $H_{0}^{l}=\sum_{\mathbf{k}, \sigma} \Psi_{\mathbf{k} \sigma}^{l, \dagger} \hat{H}_{0}^{l} \Psi_{\mathbf{k} \sigma}^{l}$ where $\hat{H}_{0}^{l}$ $\left(l=\Gamma, M_{X}, M_{Y}\right)$ is a $2 \times 2$ matrix and the spinors are defined (a)

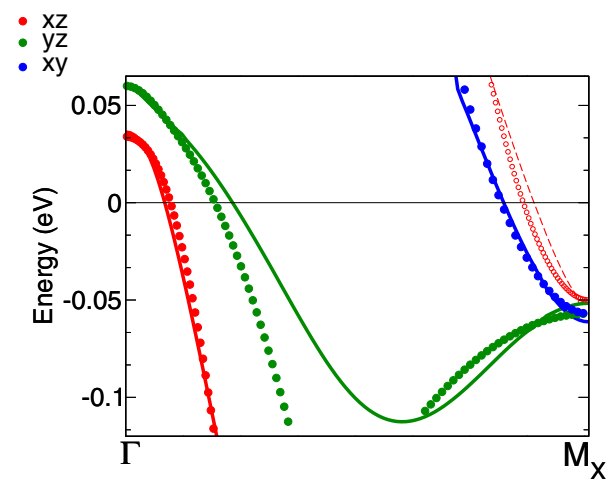

(b)

(c)
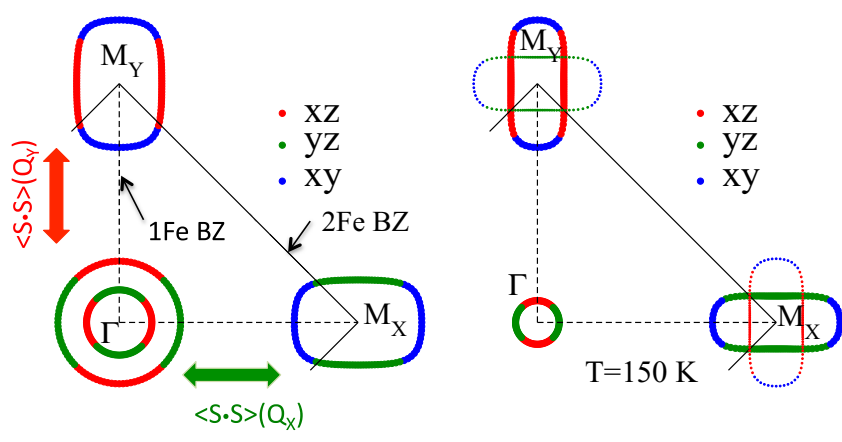

FIG. 1. General sketch of the electronic structure. (a) Lines: Band structure of FeSe at $k_{z}=0$ along the $k_{x}$ direction of the $1 \mathrm{Fe}$ BZ obtained with a tight-binding model including SOC and mass renormalization (see text). Symbols show the low-energy model that we use in the calculation [Eq. (1)]. Dashed lines and open symbols denote the bands folded at $\Gamma$ and $M_{X}$ in the $2 \mathrm{Fe}$ BZ. (b) FS cuts for the low-energy model in the $1 \mathrm{Fe} \mathrm{BZ}$. The exchange of spin fluctuations between hole and electron pockets at $M_{X}$ or $M_{Y}$ shrinks selectively the $y z$ or $x z$ orbitals, respectively. This effect, which is already present above $T_{S}$, modifies significantly the FS, as shown in panel (c) at $150 \mathrm{~K}$. Here small symbols denote the bands folded in the $2 \mathrm{Fe} \mathrm{BZ}$.

as $\Psi_{\mathbf{k} \sigma}^{\Gamma}=\left(c_{\mathbf{k} \sigma}^{y z}, c_{\mathbf{k} \sigma}^{x z}\right)$ and $\Psi_{\mathbf{k} \sigma}^{X / Y}=\left(c_{\mathbf{k} \sigma}^{y z / x z}, c_{\mathbf{k} \sigma}^{x y}\right)$. The additional $x y$ hole pocket at $\Gamma$ is not included since it is below $E_{F}$, as confirmed by previous ARPES measurements [10,11,34] and by our data at low temperature (see below). The matrix $\hat{H}^{l}$ has the general structure

$$
\hat{H}_{0}^{l}=h_{0}^{l} \tau_{0}+\vec{h}^{l} \cdot \vec{\tau}^{l},
$$

where $\vec{\tau}$ are Pauli matrices representing the orbital isospin. The bands $E_{\mathbf{k}}^{l \pm}=h_{0}^{l} \pm\left|\vec{h}^{l}\right|$ and their orbital content are then simply deduced by a straightforward diagonalization of the Hamiltonian (1). By using the explicit expressions of $\left(h_{0}, \vec{h}\right)$ detailed in the SM, one obtains the approximate band dispersions shown by symbols in Fig. 1(a) and giving the FS shown in Fig. 1(b). At the $\Gamma$ point we added explicitly to the Hamiltonian (1) the spin-orbit coupling (SOC) $\lambda[39,40]$ via the replacement of $\left|\vec{h}^{\Gamma}(\mathbf{k})\right|$ with $\sqrt{\left|\vec{h}^{\Gamma}(\mathbf{k})\right|^{2}+\lambda^{2} / 4}$. This lifts the degeneracy of the inner and outer $x z / y z$ pockets at $\Gamma$.

The basic mechanism of the FS shrinking developed in Refs. [30,31] focuses on the changes of the low-energy effective model induced by the coupling to collective modes, 
described within an Eliashberg framework via a self-energy function $\Sigma^{l}(\omega)$ for each band. The strong particle-hole asymmetry of the bands in pnictides leads to a finite real part $\zeta^{l}(\omega)$ of the self-energy $\Sigma^{l}(\omega)$, responsible for an energy-dependent shift of the interacting bands. In particular, its sign in the pocket $l$ is determined by $\zeta^{l} \simeq-\ln \left|E_{\mathrm{top}}^{l^{\prime}} / E_{\mathrm{bot}}^{l^{\prime}}\right|$, where $E_{\mathrm{top}}^{l^{\prime}}, E_{\mathrm{bot}}^{l^{\prime}}$ are the energy difference between the top/bottom of the $l^{\prime}$ pocket from the Fermi level [30]. Thus, when the exchange mechanism is interband, the sign of $\zeta^{\Gamma}(\omega)$ is controlled by the electron pockets at $M$, having $E_{\text {top }} \gg E_{\text {bot }}$, and it is thus negative. Conversely, for the hole bands $E_{\text {top }} \ll E_{\text {bot }}$ and the induced shift on the electron pockets is positive. In both cases one finds a shrinking of the FS, in agreement with observations in several iron-based pnictides $[25,26]$. The most natural bosonic mode responsible for this effect is then spin fluctuations (SF) $\langle\mathbf{S} \cdot \mathbf{S}\rangle(\mathbf{Q})$ at momenta $\mathbf{Q}_{X} \equiv \Gamma M_{X}$ or $\mathbf{Q}_{Y} \equiv \Gamma M_{Y}$ connecting hole bands at $\Gamma$ with electron bands at $M_{X}, M_{Y}$ [see Fig. 1(b)]. In this respect, our selfenergy shrinking mechanism stems somehow as a low-energy counterpart of the so-called $s^{ \pm}$Pomeranchuk instability that has been found, by renormalization-group approaches [41,42], as a possible competing instability triggered by the proximity to a spin-density wave order. Indeed, while the particle-hole asymmetry only guarantees a finite value of $\zeta^{l}(\omega)$ when the carriers are coupled to a bosonic mode, it is the interband nature of the mode, i.e., its identification with spin fluctuations, that guarantees a band shift reducing the FS areas.

So far, the FS shrinking mechanism has not been linked to the orbital degrees of freedom. To this extent, two additional ingredients should be added to the previous approach: (i) computing explicitly the self-energy effect within the orbital model (1), instead of the band model considered in Ref. [30], and (ii) accounting for the fact, pointed out recently in Ref. [32], that the mechanism of SF exchange must preserve the orbital character of the electrons. Thanks to item (ii) one can show (see SM) that the self-energy matrix $\hat{\Sigma}^{l}$, relating via the Dyson equation $\left(\hat{G}^{l}\right)^{-1}=\left(\hat{G}_{0}^{l}\right)^{-1}-\hat{\Sigma}^{l}$ the bare $\hat{G}_{0}^{l}$ and the dressed $\hat{G}^{l}$ matrix Green's functions of the model (1), simplifies considerably:

$$
\hat{\Sigma}^{\Gamma}=\left(\begin{array}{cc}
\Sigma_{y z}^{\Gamma} & 0 \\
0 & \Sigma_{x z}^{\Gamma}
\end{array}\right), \quad \hat{\Sigma}^{X / Y}=\left(\begin{array}{cc}
\Sigma_{y z / x z}^{X / Y} & 0 \\
0 & 0
\end{array}\right),
$$

where $\Sigma_{x y}^{X, Y}=0$ is a consequence of the lack of an $x y$ orbital component on the hole pockets. More importantly, we will show below that the self-energy functions select SF around precise wave vectors, linking the $y z$ orbital with SF at $Q_{X}$ and $x z$ with SF at $Q_{y}$ :

$$
\begin{aligned}
& \Sigma_{y z}^{\Gamma}(\omega), \Sigma_{y z}^{X}(\omega) \Rightarrow\langle\mathbf{S} \cdot \mathbf{S}\rangle\left(\mathbf{Q}_{X}\right), \\
& \Sigma_{x z}^{\Gamma}(\omega), \Sigma_{x z}^{Y}(\omega) \Rightarrow\langle\mathbf{S} \cdot \mathbf{S}\rangle\left(\mathbf{Q}_{Y}\right) .
\end{aligned}
$$

This result basically follows from the fact that in the $1 \mathrm{Fe} \mathrm{BZ}$, only $y z$ is present in the electron pocket at $\mathbf{Q}_{X}$, and only $x z$ is present at $\mathbf{Q}_{Y}$. Here the basic mechanism [30] controlling the sign of the self-energy corrections remains unchanged, since SF always connect hole and electron pocket. In addition, the result (3)-(4) translates the inequivalence [3] of SF at $\mathbf{Q}_{X}$ and $\mathbf{Q}_{Y}$ below $T_{S}$ into inequivalent corrections for $y z$ and $x z$ orbitals. Therefore, even though no electronic order parameter develops at $T_{s}$, neither in the charge nor in the spin sector, the anisotropy of the spin fluctuations, which is the hallmark of a spin-nematic transition, induces an anisotropy of the selfenergy corrections that acts as an effective orbital ordering. Equations (2)-(4) contain the essence of the orbital-selective shrinking that we will discuss in the following.

\section{B. Isotropic Fermi surface shrinking at $150 \mathrm{~K}$}

In Fig. 2, we present the electronic structure of FeSe measured by ARPES at $150 \mathrm{~K}$, well above the structural transition. As detailed in the SM, to observe all the bands by ARPES, it is necessary to combine even/odd light polarizations and measurements in different BZs. Three different versions of $\Gamma M$ are presented in panels (a)-(c) to cover all bands, corresponding to the three cuts indicated on the FS map (d) by thick dashed lines. The different dispersions are modeled by thin lines which are guides to the eyes, and are also reported in Fig. 2(e). The colors indicate the main orbital character (see caption).

The general structure of the bands is in agreement with the one outlined in Fig. 1. Around the $\Gamma$ point, we observe two holelike bands, made by $x z$ and $y z$ orbitals. They are split at $\Gamma$ by $20 \mathrm{meV}$, which we attribute to SOC $[10,43]$. The odd $x z / y z$ orbital (i.e., $y z$ along $k_{x}$ and $x z$ along $k_{y}$ ) forms a "saddle" band at the $M$ point, where it is expected to be degenerate with a shallow electron pocket of opposite $x z / y z$ character. Our best fit gives a small residual splitting of $\sim 5 \mathrm{meV}$ at $M$, although it could be within error bars. Note that the SOC is not effective at $M$ between these $x z$ and $y z$ bands, because they are formed by different combinations of the $2 \mathrm{Fe}$ of the unit cell [see Fig. 1(b) $[40,44]]$.

Another, deeper, electron band around $M$, appearing only in Fig. 2(b), has $x y$ character. It is often difficult to observe it in iron pnictides and was not reported before for FeSe at high temperatures. As we mentioned above, the holelike counterpart of $x y$ at $\Gamma$ is not very visible at this temperature, although it will be clearer at lower temperatures and $60 \mathrm{meV}$ below $E_{F}$ (see Fig. 3).

In Fig. 2(e), we see that the slope of the experimental dispersions (thin lines) compare well with the renormalization values assumed in Fig. 1(a) (symbols). However, the Fermi wave vectors $k_{F}$ corresponding to the $x z / y z$ orbitals are clearly too large, both for hole and electron pockets. On the contrary, it is approximately correct for the $x y$ electron band. To fully appreciate the amplitude of the shrinking, we estimate in the SM the FS volume after integration over $k_{z}$. We find that it is reduced by a factor 5 to 10 for the $x z / y z$ parts. This is the largest shrinking observed in iron-based superconductors to our knowledge. We estimated a factor 2 in Co-doped $\mathrm{BaFe}_{2} \mathrm{As}_{2}$ [26] and at most 1.2 in LiFeAs [45]. Notice that in LiFeAs there is a strong shrinking of the hole $x z / y z$ pockets, but it is compensated by an expansion of the $x y$ hole band $[45,46]$, so that the total number of carriers and the size of the electron pockets is nearly unchanged [45]. This case is, however, completely different from the shrinking considered here, involving a mutual compensation between hole and electron pockets. In addition, while the orbital redistribution of holes between the $x z / y z$ and $x y$ sheets in LiFeAs is well 
(a)

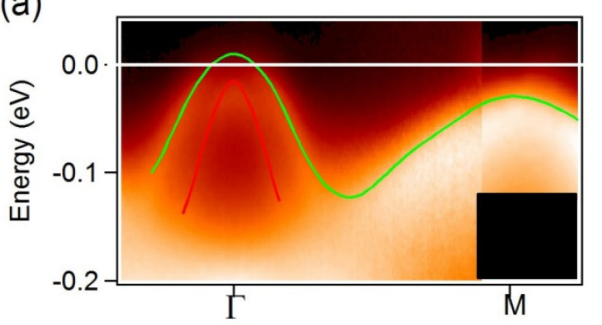

(b)

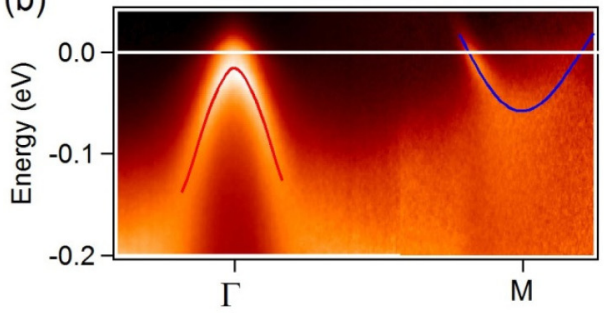

(c)

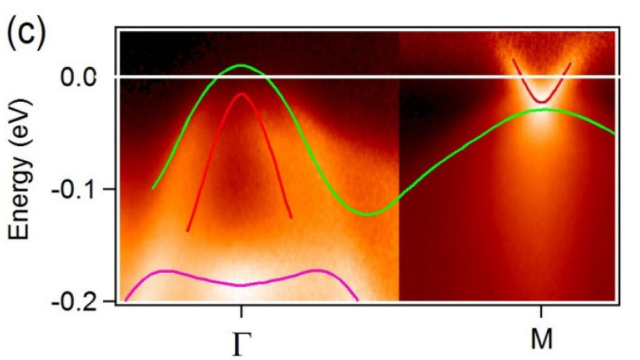

(d)

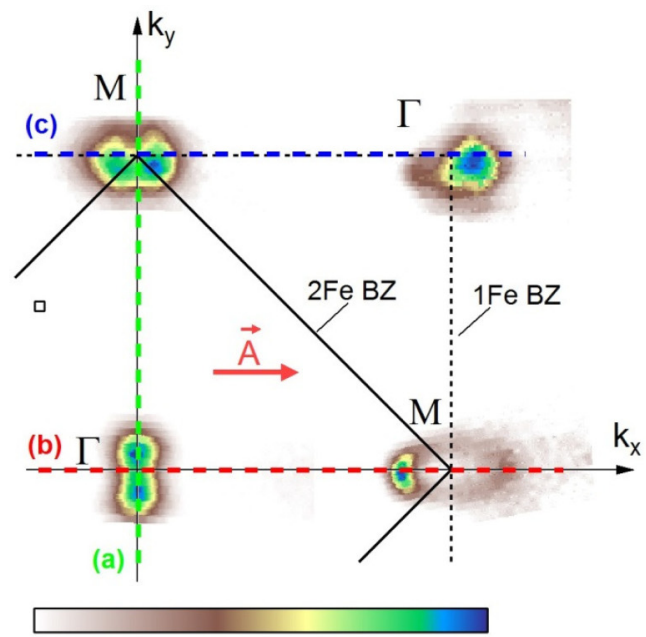

(e) 0.05
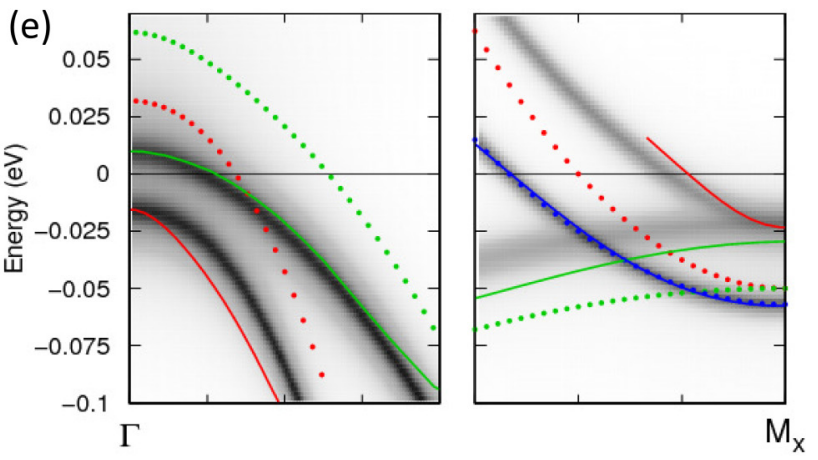

even $x z / y z$

FIG. 2. Electronic structure at $150 \mathrm{~K}$ measured by ARPES. (a)-(c) Energy momentum plots along three $\Gamma M$ directions indicated as thick dashed lines in panel (d). Lines are guides to the eyes indicating the dispersions of the different bands, with colors encoding the main orbital character (the even $x z / y z$ is $x z$ along $k_{x}$ and $y z$ along $k_{y}$ ). The data were measured at $40 \mathrm{eV}$ photon energy $\left(k_{z} \sim 0\right)$ with linear polarization along $k_{x}$. In (c), the area at each energy has been normalized to enhance the visibility of the electron pocket. (d) Fermi surface map obtained by integration of the ARPES spectral weight at $+10 \mathrm{meV}$ in a $4 \mathrm{meV}$ window. (e) Image plot in gray scale of the spectral functions of the renormalized bands at $150 \mathrm{~K}$ obtained including self-energy corrections. Ticks along abscissa correspond to $0.1 \Gamma M$. Thin lines follow the experimental data shown in (a)-(c). As in Fig 1(a), symbols indicate the bare bands of Eq. (1).

captured by DMFT [46], the same effect has not been reported by DMFT calculations in FeSe $[28,29]$.

As shown in Fig. 2(e) this orbital-selective shift of the bands is a natural outcome of the self-energy effects encoded in Eq. (2). As discussed previously [30,31], to capture the basic ingredients of the FS shrinking we can discard the full momentum dependence of the SF propagator, and use the form

$$
B_{X / Y}(\omega)=\frac{1}{\pi} \frac{\omega \omega_{0}}{\left[\omega_{s f}^{X / Y}(T)\right]^{2}+\Omega^{2}},
$$

where $\omega_{0}$ is a constant while $\omega_{s f}^{X / Y}(T)$ is the characteristic energy scale of spin modes. In the tetragonal phase we assumed the typical temperature evolution of the paramagnetic SF, $\omega_{X, Y}^{s f}(T)=\omega_{0}\left(1+T / T_{\theta}\right)$, as observed above $T_{c}$ in pnictide systems [47]. Here we used $\omega_{0} \sim 20 \mathrm{meV}$ and $T_{\theta} \sim 150 \mathrm{~K}$, in agreement with experimental results in 122 systems and more recently also in $\mathrm{FeSe}$ [21]. The self-energy functions appearing in Eqs. (2) are then computed as

$$
\begin{aligned}
& \Sigma_{y z}^{\Gamma}\left(i \omega_{n}\right)=-V T \sum_{\mathbf{k}, m} D_{X}\left(\omega_{n}-\omega_{m}\right) g_{+}^{X}\left(\mathbf{k}, i \omega_{m}\right), \\
& \Sigma_{x z}^{\Gamma}\left(i \omega_{n}\right)=-V T \sum_{\mathbf{k}, m} D_{Y}\left(\omega_{n}-\omega_{m}\right) g_{+}^{Y}\left(\mathbf{k}, i \omega_{m}\right),
\end{aligned}
$$

where $D_{X / Y}\left(\omega_{n}\right)=\int d \Omega 2 \Omega B_{X / Y}(\Omega) /\left(\Omega^{2}+\omega_{n}^{2}\right)$ is the propagator for SF along $k_{x} / k_{y}, B_{X / Y}$ is its spectral function given by Eq. (5) above, $V$ is the strength of the coupling, and $g_{ \pm}^{l}\left(\mathbf{k}, i \omega_{m}\right)$ denotes the Green's function of the $E^{l, \pm}$ band at the $l$ pocket (more details are given in the SM). Analogously for the $X, Y$ pockets one has

$$
\begin{aligned}
\Sigma_{y z}^{X}\left(i \omega_{n}\right)= & -V T \sum_{\mathbf{k}, m} D_{X}\left(\omega_{n}-\omega_{m}\right)\left[g_{+}^{\Gamma}\left(\mathbf{k}, i \omega_{m}\right)\right. \\
& \left.+g_{-}^{\Gamma}\left(\mathbf{k}, i \omega_{m}\right)\right], \\
\Sigma_{x z}^{Y}\left(i \omega_{n}\right)= & -V T \sum_{\mathbf{k}, m} D_{Y}\left[\omega_{n}-\omega_{m}\right)\left(g_{+}^{\Gamma}\left(\mathbf{k}, i \omega_{m}\right)\right. \\
& \left.+g_{-}^{\Gamma}\left(\mathbf{k}, i \omega_{m}\right)\right] .
\end{aligned}
$$


(a)

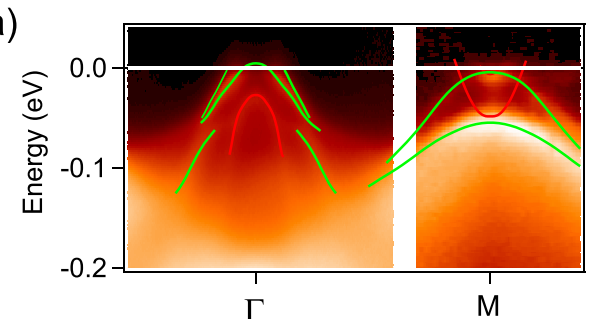

(b)

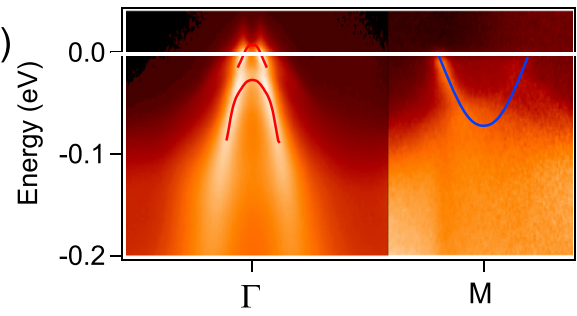

(c)

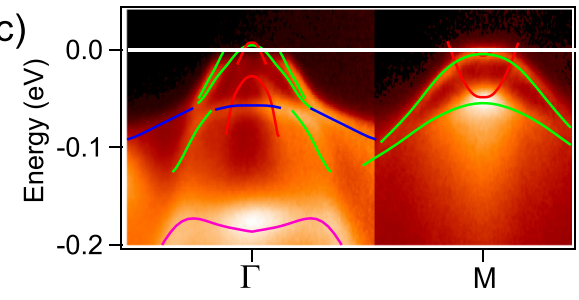

(d)

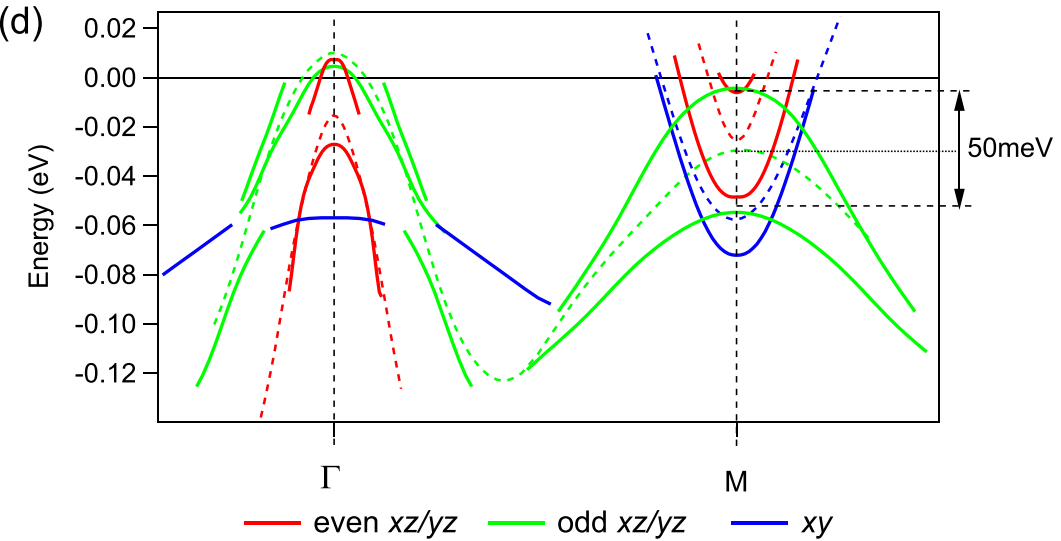

(e)
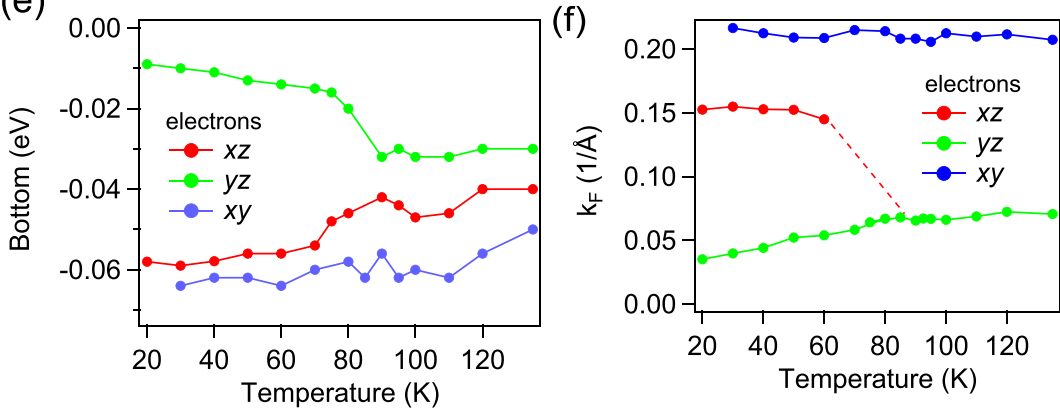

FIG. 3. Electronic structure at $20 \mathrm{~K}$ measured by ARPES. (a)-(c) Energy momentum plots in the same conditions as Fig. 2 , but at $20 \mathrm{~K}$. (d) Thin color line: experimental guides of the dispersions, also indicated in (a)-(c). Dashed lines: experimental guides at $150 \mathrm{~K}$. (e), (f) Evolution with temperature of the bottom (e) and $k_{F}$ (f) of the three electron bands.

Above $T_{S} \mathrm{SF}$ are isotropic in momentum space, i.e., $\omega_{s f}^{X}=\omega_{s f}^{Y}$, so that the self-energy corrections (3) and (4) are isotropic in the orbital space, but they have opposite signs on the hole and electron pockets [see also Figs. 5(c) and 5(d) below]. Indeed, as discussed above, the real parts of $\Sigma_{y z / x z}^{\Gamma}$ are negative, as due to the electronlike particle-hole asymmetry of the electron pockets appearing on the right-hand side of Eqs. (6) and (7), and conversely those of $\Sigma_{y z / x z}^{X / Y}$ are positive. To further account for the different degrees of nesting of the various pockets we modulated the couplings $V$ in the above equations, as detailed in the SM. Because $\Sigma_{x y}^{X / Y}=0$, as stated above, there is no shrinking on $x y$. As a consequence, while at $\Gamma$ the pockets change their size but not their shape, the electron bands become more elliptical [see Fig. 1(c)] in agreement with the experiments. We stress that because of the frequency dependence of the self-energy, the present mechanism of FS shrinking is not equivalent to a rigid band shift [31], even though this cannot be easily appreciated on the energy scale of Fig. 2(e).

\section{Anisotropic Fermi surface shrinking at $20 \mathrm{~K}$}

In Figs. 3(a)-3(c), we show the same ARPES cuts as in Fig. 2, but in the low-temperature phase, at $20 \mathrm{~K}$. As for the high-temperature case, we sketch all bands we observe by thin lines. They are also reported in Fig. 3(d), along with dashed lines representing the bands at $150 \mathrm{~K}$. The $50 \mathrm{meV}$ splitting at $20 \mathrm{~K}$ between the saddle bands at $M$ is the most dramatic feature of the nematic state and was already reported by many groups $[8-12,33,34]$.
The correct assignment of the bands at $M$ is crucial to identify which orbitals get split in the nematic phase. While the first measurements [8-12] interpreted the low-temperature data in terms of a $50 \mathrm{meV}$ splitting between the $x z$ and $y z$ orbitals, recent reports [33,34] suggested an alternative interpretation, with a large splitting between $x y$ at the bottom and a $x z / y z$ doublet at the top (whose degeneracy may be further removed by a small residual $15 \mathrm{meV}$ splitting [34]). As we clearly separate $x z / y z$ and $x y$ at all temperatures (see Fig. 4), the distinction is easier in our case. Figure 4(b) shows that there is little change of the $x y$ electron band as a function of temperature, except for a small deepening of the band bottom at low temperatures, which we attribute to a reduction of the renormalization from 5 to 4.2 (see SM). Apart from this effect, the size of the pockets barely changes with temperature, showing that this band does not participate actively to the shrinking mechanism. We report the experimental shape of the $x y$ electron band on Fig. 4(a) as a dotted line. As the bottom of the $x y$ electron band should be degenerate with the $x y$ saddle band (at least for high temperatures; more details are given in the SM), the saddle band we observe moving down has to be $y z$, clearly supporting the existence of a large nematic splitting of the $x z / y z$ orbitals. The temperature evolution of the spectra at $M$ is summarized in Figs. 3(e) and 3(f), where we report the temperature dependence of the bottom of the bands at $M$ and of their $k_{F}$. The three different $k_{F}$ 's at low temperatures are only consistent with the situation indicated in Fig. 3(d).

Around $\Gamma$ the orbital splitting is less obvious, since the experimental data of Fig. 3(d) at 20 and $150 \mathrm{~K}$ largely overlap. This effect led some authors to conclude that there is no or 
(a) $\mathrm{M} \Gamma_{2}$, odd detection
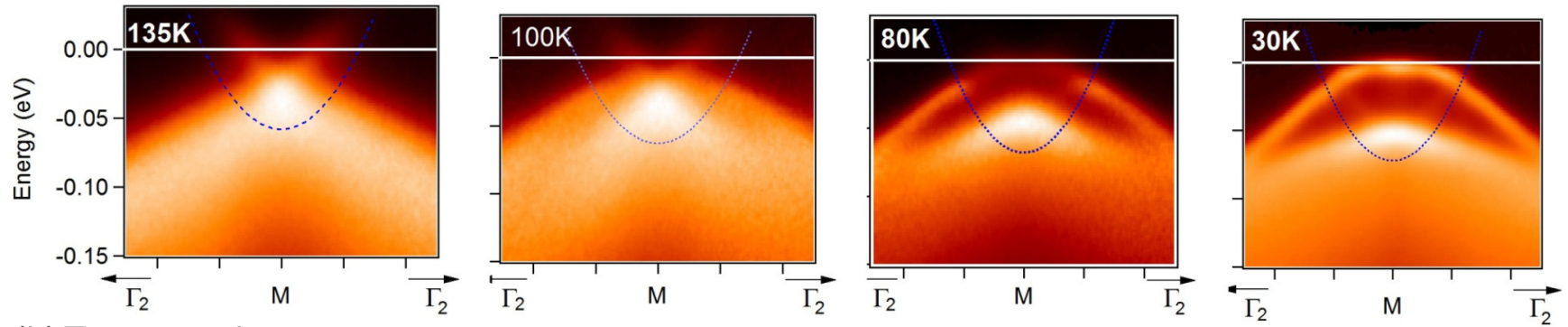

(b) $\Gamma_{1} \mathrm{M}$, even detection
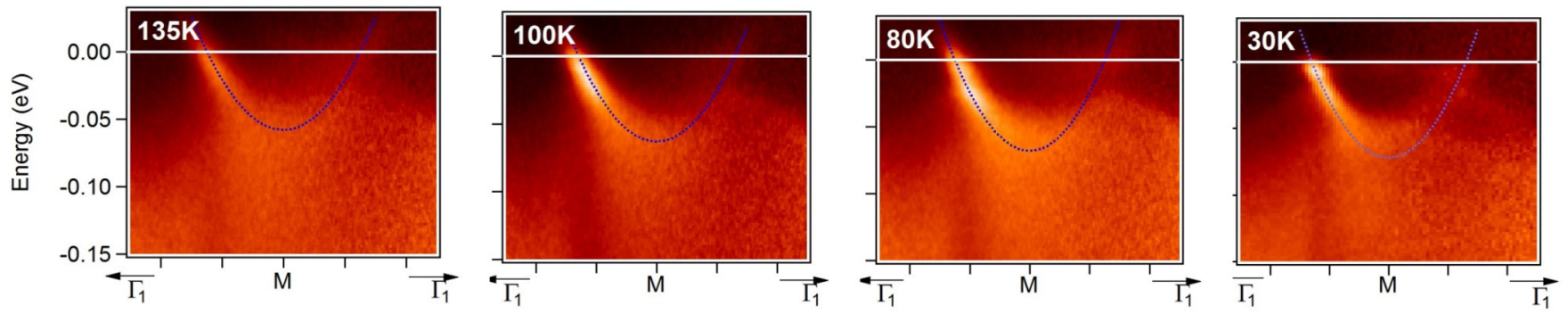

FIG. 4. Temperature evolution at $M$ point. (a) Energy-momentum plots around $M$ in the same conditions as Fig. 2(c), but with all energy distribution curves normalized to constant area, which emphasizes the saddle bands. (b) Energy-momentum plots around $M$ in the same conditions as Fig. 2(b), showing the $x y$ electron band. The fit of this band (dotted blue line) is reported in (a). $\Gamma_{1}$ and $\Gamma_{2}$ are the $\Gamma_{\text {points at }}$ $(0,0)$ and $(\pi, \pi)$, respectively.

little change there $[11,12]$. However, there are some changes such as the appearance of a characteristic "hat" on top of the inner band in Fig. 3(b). As was also concluded in Ref. [13], we will argue below that it is due to a SOC-avoided crossing of the two hole bands along $k_{x}$, which implies sizable shifts of the $x z / y z$ bands at $\Gamma$ also.

The observed modifications of the Fermi surface can be very well understood within our orbital-selective spin-fluctuations scenario. Indeed, the SF are expected to become inequivalent below $T_{S}$, as usually assumed for a spin-driven nematic transition [3], making SF stronger at the wave vector $\mathbf{Q}_{X}$ where antiferromagnetic order usually develops at lower temperature. Even though long-range magnetic order is not observed in FeSe, SF have been detected [20-22] and we assume that they become anisotropic below $T_{S}$, with a softening $\omega_{s f}^{X}<\omega_{s f}^{Y}$ that is the hallmark of having $\left.\langle\mathbf{S} \cdot \mathbf{S}\rangle\left(\mathbf{Q}_{X}\right)\right\rangle\langle\mathbf{S} \cdot \mathbf{S}\rangle\left(\mathbf{Q}_{Y}\right)$, as sketched in Figs. 5(a) and 5(b). This has the immediate effect, from Eqs. (3) and (4), to split the self-energy corrections of the $x z$ and $y z$ orbitals, making in general their absolute values larger for $y z$. Taking into account the different sign of the self-energy shifts (6)-(7) and (8)-(9), respectively at the hole and electron pockets, one immediately finds that $\Delta \zeta^{\Gamma} \equiv \zeta_{x z}^{\Gamma}-$ $\zeta_{y z}^{\Gamma}>0$, while $\Delta \zeta^{M}<0$, as calculated in Figs. 5(c)-5(e). This is in agreement with previous experiments in detwinned samples $[8,13]$.

In Fig. 6, we report the theoretically computed spectral functions along $\Gamma M_{X}$ and $\Gamma M_{Y}$ and the FS. They are compared with our experimental data, where we have used the information from detwinned experiments $[8,13]$ and the above band assignment to determine which data lines correspond to measurements along $\Gamma M_{X}$ or $\Gamma M_{Y}$. Below $T_{S}$ the orbitaldependent shrinking induces an elliptical deformation of the hole pocket, which acquires mainly $x z$ character. At
$M_{X}$, it shrinks the $y z$ orbital further, while at $M_{Y}$, the $x z$ orbital expands back. In order to reproduce quantitatively the experimental data, we computed the spectral functions of Fig. 6 by using as a fitting parameter the splitting of the SF energies $\omega_{s f}^{X / Y}$ (details are given in the SM). Very good agreement is obtained with the parameters of Fig. 5. Note that the SF-induced splitting at $\Gamma(30 \mathrm{meV})$ is comparable to the one at $M(-40 \mathrm{meV})$, evidencing that its effect on the
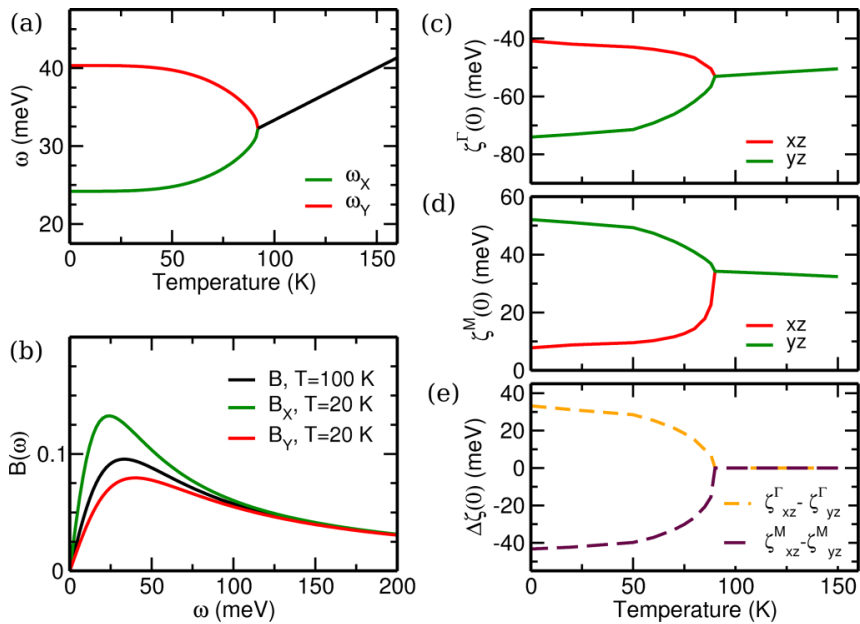

FIG. 5. Evolution of the spin fluctuation and nematic splitting with temperature. (a) Temperature evolution of the SF energies $\omega_{s f}^{X / Y}$ across the nematic transition. The SF propagator (5) becomes anisotropic below $T_{S}$, as shown in panel (b). (c)-(e) The anisotropy of the SF below $T_{S}$ induces a nematic splitting of the self-energy corrections for the $x z$ and $y z$ orbitals. Their real parts at zero frequency $\zeta(\omega=0)$ are shown in panels (c) and (d) for the $\Gamma, M$ pockets. The resulting $x z / y z$ splitting below $T_{S}$ is shown in (d). 
(a)

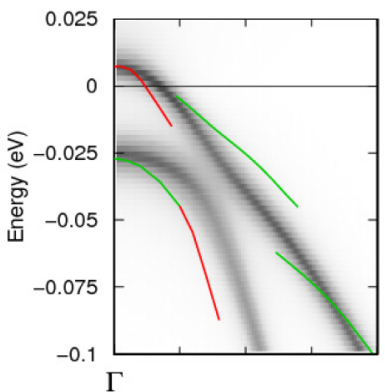

(b)

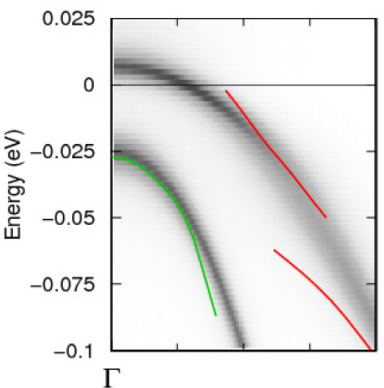

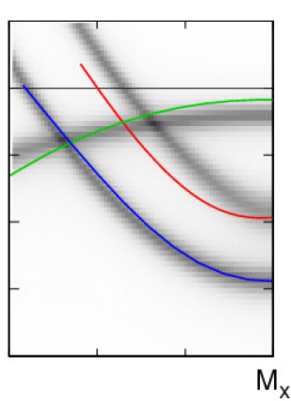

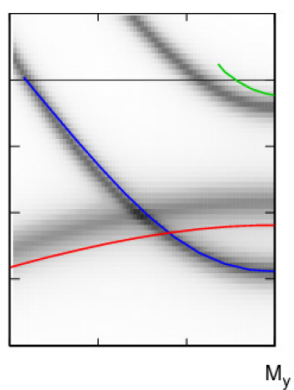

(c)
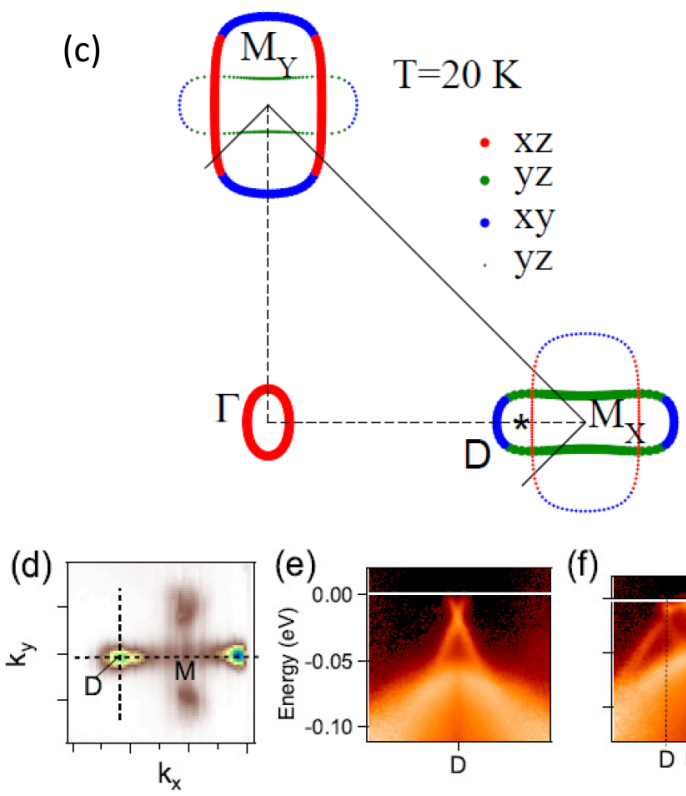

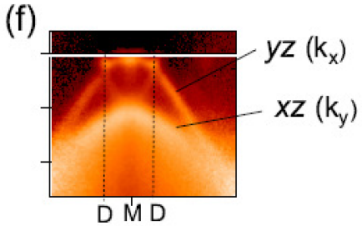

FIG. 6. Electronic structure in the nematic state modeled in the spin-fluctuation scenario. Spectral functions of the renormalized bands at $20 \mathrm{~K}$ along $\Gamma M_{X}$ (a) and $\Gamma M_{Y}$ (b). Ticks along the abscissa correspond to $0.1 \Gamma M$. Thin lines reproduce the experimental data detailed in Fig. 3. The "break" at $-60 \mathrm{meV}$ in the dispersion of the outer hole band is due to the crossing of the $x y$ hole band. Along $k_{x}$ the main orbital character switches from $y z$ to $x z$ on the inner band and from $x z$ to $y z$ on the outer band, due to the crossing of the two bands hybridized through SOC. (c) Fermi surface calculated for low temperatures. (d) Map of the ARPES spectral weight integrated around $M$ at -15 meV over $4 \mathrm{meV}$ and dispersions in the two perpendicular dispersions across the Dirac point $D$. (e), (f) Energy-momentum plots in two perpendicular cuts across the Dirac point.

overall band structure at $\Gamma$ is less apparent only because it is hidden by the strong SOC present there. We can then see from Fig. 5(b) that the resulting anisotropy of the spin modes below $T_{s}$ is relatively weak, which is compatible with experiments in twinned samples [20-22]. Nonetheless, the effects on the band dispersions are quite strong. This is due to the particularly large particle-hole asymmetry of the bands in FeSe, resulting first from the strong renormalization inherent to FeSe and second from the high temperature shrinking.

The changes of the FS structure that we discussed so far are dictated by the real part of the SF-induced self-energy corrections. In addition, the imaginary part of the self-energy also becomes orbital dependent below $T_{S}$ and determines the lifetime of carriers in the nematic phase. Indeed, the dispersions shown in Figs. 6(e) and 6(f) suggest very different properties along $k_{x}$ and $k_{y}$ in the nematic state. Along $k_{x}$, a Dirac cone is formed between $x y$ and the $y z$ saddle band, just $15 \mathrm{meV}$ below $E_{F}[48,49]$. The lines forming the Dirac cone are remarkably narrow, contrasting with the much broader lines belonging to the perpendicular domain, even for similar binding energies. Along $k_{y}$, the electron pocket, although enlarged, is made out of bands that appear very incoherent in ARPES. This coexistence of coherent and incoherent carriers is a very important and unusual characteristic of the metallic state of FeSe at low temperatures.

\section{CONCLUSION}

The dichotomy between the orbital-driven and spin-driven scenarios discussed so far for pnictides is connected to the difference between "hard" and "soft" possible realizations of electron nematicity. In the former case the nematic transition breaks explicitly a symmetry with the emergence of a finite electronic order parameter, which directly affects the singleparticle electronic properties probed by ARPES. In the latter case, instead the symmetry is broken only at the level of collective electronic fluctuations, which naturally affect collective two-particle properties, as probed, e.g., by optical or Raman spectroscopy [50-52]. For this reason the strong modification of the electronic structure below $T_{S}$ seen by ARPES in FeSe triggered the idea [53,54] that a hard orbital order is needed to explain the modification of the electronic structure. However, while it is indeed consistent with the experimental observations, its justification at the microscopic level requires a specific fine-tuning of the interactions $[17,41,55]$. On the other hand, in pnictides also collective fluctuations can affect the single-particle properties, and modify the Fermi surface [30]. This mechanism, which relies on quite general conditions (the exchange of spin fluctuations between hole and electron bands in pnictides), is already operative at high temperatures, and it naturally leads to the observed FS evolution in the nematic state. Thus, the orbital-selective spin-fluctuations scenario explains why a soft nematic transition can give rise, thanks to the strong spin-orbit entanglement, to an order-parameter-like behavior of the electronic structure, even in the absence of any hard symmetry breaking. Besides reconciling different views, our results revise the standard paradigms for the understanding of hard and soft nematicity in the collective electronic behavior.

\section{ACKNOWLEDGMENTS}

V.B. and J.M. acknowledge financial support from the ANR "PNICTIDES.” L.B. acknowledges financial support by Italian 
MIUR under projects FIRB-HybridNanoDev-RBFR1236VV, PRINRIDEIRON-2012X3YFZ2, and Premiali-2012 ABNANOTECH. B.V. acknowledges funding from Ministerio de
Economía y Competitividad (Spain) via Grants No. FIS201453219-P, No. FIS2011-29689, and Fundación Ramón Areces.
[1] E. Fradkin, S. Kivelson, M. Lawler, J. Eisenstein, and A. Mackenzie, Ann. Rev. Condens. Matter Phys. 1, 153 (2010).

[2] I. R. Fisher, L. Degiorgi, and Z. Shen, Rep. Prog. Phys. 74, 124506 (2011).

[3] R. M. Fernandes, A. V. Chubukov, and J. Schmalian, Nat. Phys. 10, 97 (2014).

[4] Y. Gallais and I. Paul, C. R. Phys. 17, 113 (2016).

[5] A. E. Böhmer, F. Hardy, F. Eilers, D. Ernst, P. Adelmann, P. Schweiss, T. Wolf, and C. Meingast, Phys. Rev. B 87, 180505 (2013).

[6] F.-C. Hsu, J.-Y. Luo, K.-W. Yeh, T.-K. Chen, T.-W. Huang, P. M. Wu, Y.-C. Lee, Y.-L. Huang, Y.-Y. Chu, D.-C. Yan, and M.-K. Wu, Proc. Natl. Acad. Sci. USA 105, 14262 (2008).

[7] M. A. Tanatar, A. E. Böhmer, E. I. Timmons, M. Schütt, G. Drachuck, V. Taufour, K. Kothapalli, A. Kreyssig, S. L. Bud'ko, P. C. Canfield, R. M. Fernandes, and R. Prozorov, Phys. Rev. Lett. 117, 127001 (2016).

[8] T. Shimojima, Y. Suzuki, T. Sonobe, A. Nakamura, M. Sakano, J. Omachi, K. Yoshioka, M. Kuwata-Gonokami, K. Ono, H. Kumigashira, A. E. Bohmer, F. Hardy, T. Wolf, C. Meingast, H. v. Lohneysen, H. Ikeda, and K. Ishizaka, Phys. Rev. B 90, 121111 (2014).

[9] K. Nakayama, Y. Miyata, G. N. Phan, T. Sato, Y. Tanabe, T. Urata, K. Tanigaki, and T. Takahashi, Phys. Rev. Lett. 113, 237001 (2014).

[10] M. D. Watson, T. K. Kim, A. A. Haghighirad, N. R. Davies, A. McCollam, A. Narayanan, S. F. Blake, Y. L. Chen, S. Ghannadzadeh, A. J. Schofield, M. Hoesch, C. Meingast, T. Wolf, and A. I. Coldea, Phys. Rev. B 91, 155106 (2015).

[11] P. Zhang, T. Qian, P. Richard, X. P. Wang, H. Miao, B. Q. Lv, B. B. Fu, T. Wolf, C. Meingast, X. X. Wu, Z. Q. Wang, J. P. Hu, and H. Ding, Phys. Rev. B 91, 214503 (2015).

[12] Y. Zhang et al., Phys. Rev. B 94, 115153 (2016).

[13] Y. Suzuki, T. Shimojima, T. Sonobe, A. Nakamura, M. Sakano, H. Tsuji, J. Omachi, K. Yoshioka, M. Kuwata-Gonokami, T. Watashige, R. Kobayashi, S. Kasahara, T. Shibauchi, Y. Matsuda, Y. Yamakawa, H. Kontani, and K. Ishizaka, Phys. Rev. B 92, 205117 (2015).

[14] W. Lv, J. Wu, and P. Phillips, Phys. Rev. B 80, 224506 (2009).

[15] Y. Su, H. Liao, and T. Li, J. Phys.: Condens. Matter 27, 105702 (2015).

[16] S. Mukherjee, A. Kreisel, P. J. Hirschfeld, and B. M. Andersen, Phys. Rev. Lett. 115, 026402 (2015).

[17] K. Jiang, J. Hu, H. Ding, and Z. Wang, Phys. Rev. B 93, 115138 (2016).

[18] S. Baek, D. Efremov, J. M. Ok, J. S. Kim, J. van den Brink, and B. Buchner, Nat. Mater. 14, 210 (2014).

[19] A. E. Böhmer, T. Arai, F. Hardy, T. Hattori, T. Iye, T. Wolf, H. v. Löhneysen, K. Ishida, and C. Meingast, Phys. Rev. Lett. 114, 027001 (2015).

[20] Q. Wang, Y. Shen, B. Pan, Y. Hao, M. Ma, F. Zhou, P. Steffens, K. Schmalzl, T. R. Forrest, M. Abdel-Hafiez, X. Chen, D. A. Chareev, A. N. Vasiliev, P. Bourges, Y. Sidis, H. Cao, and J. Zhao, Nat. Mater. 15, 159 (2016).
[21] M. C. Rahn, R. A. Ewings, S. J. Sedlmaier, S. J. Clarke, and A. T. Boothroyd, Phys. Rev. B 91, 180501 (2015).

[22] S. Shamoto, K. Matsuoka, R. Kajimoto, M. Ishikado, T. Watashige, S. Kasahara, M. Nakamura, H. Kontani, T. Shibauchi, and Y. Matsuda, arXiv:1511.04267.

[23] C. W. Luo, P. C. Cheng, S.-H. Wang, J.-C. Chiang, J.-Y. Lin, K. H. Wu, J. Y. Juang, D. A. Chareev, O. S. Volkova, and A. N. Vasiliev, arXiv:1603.08710.

[24] J. K. Glasbrenner, I. I. Mazin, H. O. Jeschke, P. J. Hirschfeld, R. M. Fernandes, and R. Valentí, Nat. Phys. 11, 953 (2015).

[25] A. I. Coldea, J. D. Fletcher, A. Carrington, J. G. Analytis, A. F. Bangura, J.-H. Chu, A. S. Erickson, I. R. Fisher, N. E. Hussey, and R. D. McDonald, Phys. Rev. Lett. 101, 216402 (2008).

[26] V. Brouet, P.-H. Lin, Y. Texier, J. Bobroff, A. Taleb-Ibrahimi, P. Le Fèvre, F. Bertran, M. Casula, P. Werner, S. Biermann, F. Rullier-Albenque, A. Forget, and D. Colson, Phys. Rev. Lett. 110, 167002 (2013).

[27] L. de' Medici, G. Giovannetti, and M. Capone, Phys. Rev. Lett. 112, 177001 (2014)

[28] M. Aichhorn, S. Biermann, T. Miyake, A. Georges, and M. Imada, Phys. Rev. B 82, 064504 (2010).

[29] Z. P. Yin, K. Haule, and G. Kotliar, Phys. Rev. B 86, 195141 (2012).

[30] L. Ortenzi, E. Cappelluti, L. Benfatto, and L. Pietronero, Phys. Rev. Lett. 103, 046404 (2009).

[31] L. Benfatto and E. Cappelluti, Phys. Rev. B 83, 104516 (2011).

[32] L. Fanfarillo, A. Cortijo, and B. Valenzuela, Phys. Rev. B 91, 214515 (2015).

[33] M. D. Watson, T. K. Kim, L. C. Rhodes, M. Eschrig, M. Hoesch, A. A. Haghighirad, and A. I. Coldea, arXiv:1603.04545.

[34] A. Fedorov, A. Yaresko, T. K. Kim, E. Kushnirenko, E. Haubold, T. Wolf, M. Hoesch, A. Grueneis, B. Buechner, and S. V. Borisenko, arXiv:1606.03022.

[35] S. Karlsson, P. Strobel, A. Sulpice, C. Marcenat, M. Legendre, F. Gay, S. Pairis, O. Leynaud, and P. Toulemonde, Supercond. Sci. Technol. 28, 105009 (2015).

[36] A. Audouard, F. Duc, L. Drigo, P. Toulemonde, S. Karlsson, P. Strobel, and A. Sulpice, Europhys. Lett. 109, 27003 (2015).

[37] See Supplemental Material at http://link.aps.org/supplemental/ 10.1103/PhysRevB.94.155138 for further information on comparison with band structure calculations, geometry of ARPES measurements and theoretical model are given.

[38] H. Eschrig and K. Koepernik, Phys. Rev. B 80, 104503 (2009).

[39] V. Cvetkovic and O. Vafek, Phys. Rev. B 88, 134510 (2013).

[40] R. M. Fernandes and O. Vafek, Phys. Rev. B 90, 214514 (2014).

[41] H. Zhai, F. Wang, and D.-H. Lee, Phys. Rev. B 80, 064517 (2009).

[42] A. V. Chubukov, M. Khodas, and R. M. Fernadnez, arXiv: 1602.05503.

[43] J. Maletz, V. B. Zabolotnyy, D. V. Evtushinsky, S. Thirupathaiah, A. U. B. Wolter, L. Harnagea, A. N. Yaresko, A. N. Vasiliev, 
D. A. Chareev, A. E. Bohmer, F. Hardy, T. Wolf, C. Meingast, E. D. L. Rienks, B. Buchner, and S. V. Borisenko, Phys. Rev. B 89, 220506 (2014).

[44] N. Hao and J. Hu, Phys. Rev. X 4, 031053 (2014).

[45] V. Brouet, D. LeBoeuf, P.-H. Lin, J. Mansart, A. Taleb-Ibrahimi, P. Le Fevre, F. Bertran, A. Forget, and D. Colson, Phys. Rev. B 93, 085137 (2016).

[46] G. Lee, H. S. Ji, Y. Kim, C. Kim, K. Haule, G. Kotliar, B. Lee, S. Khim, K. H. Kim, K. S. Kim, K.-S. Kim, and J. H. Shim, Phys. Rev. Lett. 109, 177001 (2012).

[47] D. S. Inosov, J. T. Park, P. Bourges, D. L. Sun, Y. Sidis, A. Schneidewind, K. Hradil, D. Haug, C. T. Lin, B. Keimer, and V. Hinkov, Nat. Phys. 6, 178 (2010).

[48] S. Y. Tan, Y. Fang, D. H. Xie, W. Feng, C. H. P. Wen, Q. Song, Q. Y. Chen, W. Zhang, Y. Zhang, L. Z. Luo, B. P. Xie, X. C. Lai, and D. L. Feng, Phys. Rev. B 93, 104513 (2016).
[49] W. Li, Y. Zhang, J. J. Lee, H. Ding, M. Yi, Z. Li, P. Deng, K. Chang, S.-K. Mo, M. Hashimoto, D. H. Lu, X. Chen, R. G. Moore, Q.-K. Xue, and Z.-X. Shen, arXiv:1509.01892.

[50] Y. Gallais, R. M. Fernandes, I. Paul, L. Chauvière, Y.-X. Yang, M.-A. Méasson, M. Cazayous, A. Sacuto, D. Colson, and A. Forget, Phys. Rev. Lett. 111, 267001 (2013).

[51] F. Kretzschmar, T. Bohm, U. Karahasanovic, B. Muschler, A. Baum, D. Jost, J. Schmalian, S. Caprara, M. Grilli, C. Di Castro, J. G. Analytis, J. H. Chu, I. R. Fisher, and R. Hackl, Nat. Phys. 12, 560 (2016).

[52] V. K. Thorsmolle, M. Khodas, Z. P. Yin, C. Zhang, S. V. Carr, P. Dai, and G. Blumberg, Phys. Rev. B 93, 054515 (2016).

[53] A. Kreisel, S. Mukherjee, P. J. Hirschfeld, and B. M. Andersen, Phys. Rev. B 92, 224515 (2015).

[54] Y. Liang, X. Wu, and J. Hu, Chin. Phys. Lett. 32, 117402 (2015).

[55] S. Onari, Y. Yamakawa, and H. Kontani, Phys. Rev. Lett. 116, 227001 (2016). 